\section{Roles of Bifocal, Homer, and F-actin in anchoring Oskar to the posterior cortex of Drosophila oocytes}

\author{
Kavita Babu, ${ }^{1,2,3}$ Yu Cai, ${ }^{2}$ Sami Bahri, ${ }^{2}$ \\ Xiaohang Yang, ${ }^{2}$ and William Chia ${ }^{1,4}$ \\ ${ }^{1}$ MRC Centre for Developmental Neurobiology, King's \\ College London, London SE1 1UL, UK; ${ }^{2}$ Institute of \\ Molecular and Cell Biology, Singapore 117609
}

Transport, translation, and anchoring of osk mRNA and proteins are essential for posterior patterning of Drosophila embryos. Here we show that Homer and Bifocal act redundantly to promote posterior anchoring of the osk gene products. Disruption of actin microfilaments, which causes delocalization of Bifocal but not Homer from the oocyte cortex, severely disrupts anchoring of osk gene products only when Homer (not Bifocal) is absent. Our data suggest that two processes, one requiring Bifocal and an intact F-actin cytoskeleton and a second requiring Homer but independent of intact F-actin, may act redundantly to mediate posterior anchoring of the osk gene products.

Supplemental material is available at http://www.genesdev.org.

Received August 13, 2003; revised version accepted November $26,2003$.

Asymmetric localization of determinants to specific subcellular compartments of germline and somatic cells plays important roles in pattern formation and cell-fate specification. Localization of the maternal determinant Oskar to the posterior pole of Drosophila oocytes is critical for posterior patterning and pole plasm assembly (Lehmann and Nusslein-Volhard 1986; Ephrussi and Lehmann 1992). Spatial restriction of Oskar depends on transport- and localization-dependent translation of oskar mRNA, as well as anchoring of osk mRNA and proteins to the posterior cortex (Ephrussi et al. 1991; Kim-Ha et al. 1991; Rongo et al. 1995; Gunkel et al. 1998). Microtubules and associated motors are required for posterior transport of osk mRNA, and microfilament and actin binding proteins have been implicated in the anchoring of the osk gene products (Erdelyi et al. 1995; Glotzer et al. 1997; Brendza et al. 2000; Saxton 2001; Jankovics et al. 2002; Polesello et al. 2002).

Drosophila Homer (Hom), the Drosophila homolog of the vertebrate Homer (Brakeman et al. 1997) is an F-actin binding protein (Shiraishi et al. 1999). Mutants in hom

[Keywords: Bifocal; Homer; Oskar; F-actin; anchoring]

${ }^{3}$ Present address: Temasek Life Sciences Laboratory, 1 Research Link, NUS, Singapore 117604

${ }^{4}$ Corresponding author.

E-MAIL william.chia@kcl.ac.uk; FAX 44-207-8486550.

Article and publication are at http://www.genesdev.org/cgi/doi/10.1101/ gad.282604. show defects in locomotor activity and mating behavior (Diagana et al. 2002). Bifocal (Bif) is a novel protein which was shown to colocalize with F-actin and is required for normal photoreceptor rhabdomere formation and axon guidance (Helps et al. 2001; Ruan et al. 2002). Bif was shown to form a complex with both F-actin and microtubules in vitro (Sisson et al. 2000). Total loss of function of bif or hom are viable and fertile with no obvious embryonic defects (Bahri et al. 1997; Diagana et al. 2002). Here we show that simultaneous loss of Hom (Diagana et al. 2002) and Bif (Bahri et al. 1997), while not affecting osk mRNA transport and translation, delocalizes osk RNA and proteins from the posterior cortex. Latrunculin A disruption of actin microfilaments, which causes delocalization of Bif but not Hom from the posterior cortex of wild-type oocytes, causes only minor defects in the anchoring of osk gene products. However, Latrunculin A disruption in the absence of Hom, but not in the absence of Bif, causes severe defects in posterior anchoring of osk RNA and proteins. Our data suggest that two processes, one that requires Bif and an intact F-actin cytoskeleton and a second process requiring Hom but independent of an intact F-actin cytoskeleton, may act redundantly to mediate posterior anchoring of the osk gene products.

\section{Results and Discussion}

bif;hom double mutants show severe defects in the anchoring of osk RNA and proteins

Both Bif and Hom show asymmetric localization at the apical cortex of embryonic neuroblasts (data not shown), indicating that these F-actin binding proteins may be involved in neuroblast asymmetric divisions. However, animals lacking both the maternal and zygotic components of either gene are fertile, viable, and show no obvious defects in embryonic CNS development. This prompted us to make double mutants of bif and hom. However, although double homozygous mutant females are viable, they show defects in oogenesis, with the vast majority of the eggs produced remaining unfertilized, as judged by the lack of staining in eggs using an antibody directed against the sperm tail (data not shown; Karr 1991; Graner et al. 1994). In the few fertilized embryos which do undergo development, the numbers of Vasapositive germ cells are drastically reduced (from $\sim 33$ in wild type, $\mathrm{n}=15$; to seven in double mutants, $\mathrm{n}=12$; data not shown), suggesting possible defects in the function or localization of posterior determinants during oogenesis.

Analyses of bif and hom single mutants as well as double mutant oocytes indicate that the two genes act in a redundant manner for the correct anchoring of posteriorly but not anteriorly localized molecules. In stage 10 oocytes, posterior group molecules, including oskar (osk) RNA, the two isoforms of Osk proteins (Rongo et al. 1995), Staufen (Stau; St Johnston et al. 1991), and a fusion protein in which $\beta$-galactosidase has been fused to the $\mathrm{N}$-terminal extension of the long form of the Osk protein (referred to as Osk- $\beta$ Gal) used as a marker for the long form of the Osk protein, which has been shown to have a role in the posterior cortical maintenance of Osk 

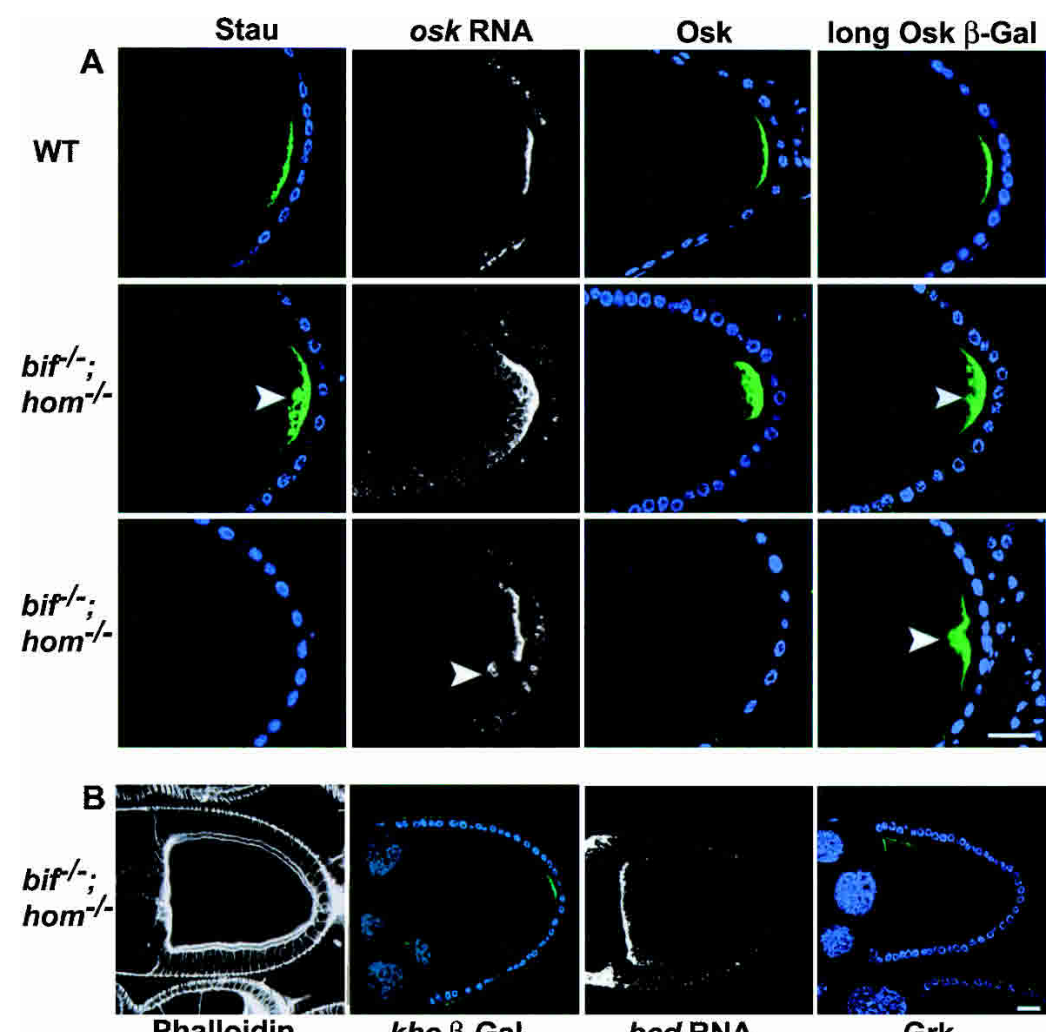

bcd RNA
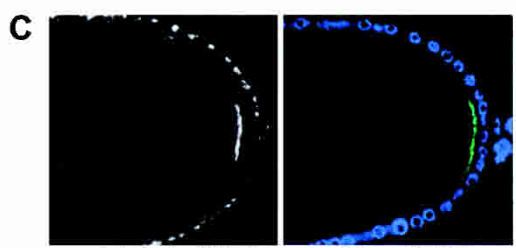

Stau (WT)
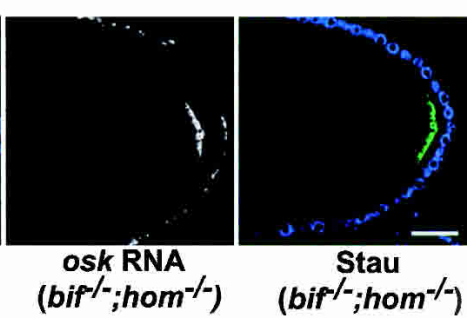

Figure 1. Loss of both bifocal and homer causes defective anchoring of the posterior determinants in oocytes. (A) Stage 10 wild-type and bif;hom double mutant oocytes showing the localization of Stau (green), osk RNA (white), Osk (green), and Osk- $\beta$ Gal (green). Two sets of double mutant oocytes are shown (second and third rows) to illustrate the different severity of the phenotypes observed; for example, Stau and Osk can either be loosely cytoplasmic (second row) or undetectable (third row). Arrowheads point to sites of cytoplasmic localization. DNA staining (blue) marks the position of follicle cell nuclei. (B) Double mutant oocytes assessed with phalloidin, $k h c \beta-G a l, b c d$ RNA in situ, and anti-Grk, all of which appear normal. (C) osk RNA and Stau localization in wild-type and double mutant stage 9 oocytes; the normal localization seen at stage 9 indicates that the defects seen in stage 10 double mutant oocytes are due to defects in anchoring. (Bar length is $10 \mu \mathrm{m}$ in all figures.)

(Gunkel et al. 1998; Vanzo and Ephrussi 2002), are all localized as tight posterior cortical crescents in wildtype oocytes (Fig. 1A). In most double mutant oocytes, these molecules, when detectable, are present largely at the posterior region; however in contrast to wild-type oocytes, they show a diffuse distribution that extends into regions of the posterior cytoplasm distinctly interior to the posterior cortex (Fig. 1A). In about $30 \%$ of the cases, Osk or Stau protein cannot be detected (Fig. 1A; see Supplemental Table 1 for quantitation). The defects seen in the oocytes of double mutants are essentially absent in the single mutant oocytes. These findings indicate that whereas bif and hom are individually dispensable, together they are required for the localization of the posterior components of the oocytes. These defects in localization are specific for the posterior group molecules, because the anterior/dorsal localization of Gurken (Neuman-Silberberg and Schupbach 1996) and anterior localization of bicoid RNA (Berleth et al. 1988) are unaffected (Fig. 1B).

Not surprisingly, staining with anti-Bif (Helps et al. 2001) and anti-Hom antibodies indicates that both proteins are expressed in the oocyte. Bif localizes to the oocyte cortex in a manner very similar to that seen for F-actin (Fig. 2A). The staining seen with the anti-Hom antibody is highly punctated and, although localization is cortically enriched, Hom is also present in the cytoplasm (Fig. 2B). The cortical staining seen in wild-type oocytes (and nurse cells) is absent in mutant oocytes stained with the corresponding antibodies, confirming the specificity of both antibodies and that the mutant alleles do not produce detectable amounts of protein (Fig. 2C). As hom ${ }^{L L 17}$ is a complete deletion of the coding region and $b i f^{R 47}$ removes a significant portion of the coding region (Bahri et al. 1997), they are both likely to be null alleles.

Several observations indicate that the defect in posterior localization of the osk gene products is due to defective anchoring and not transport or translation of osk RNA. Osk RNA and Osk proteins as well as Stau are localized normally in stage 9 double mutant oocytes (Fig. 1C; data not shown). Consistent with this, both the F-actin and microtubule cytoskeletons in the double mutants are indistinguishable from those in the wild-type oocytes (Fig. 1B; data not shown). Not only does the polarity of the microtubules appear normal, as assayed using a kinesin heavy chain (khc) $\beta-\mathrm{Gal}$ marker (Fig. 1B; Clark et al. 1994; Brendza et al. 2000), the cytoskeleton-dependent cytoplasmic streaming (Theurkauf 1994) is also absent in stage 9 oocytes and occurs normally in stage 10 double mutant oocytes, as is seen with wild-type oocytes (data not shown). Taken together, these observations indicate that the double mutant oocytes retain, at a gross level, normal cytoskeletal structure. They can transport osk mRNA to the posterior cortex, and translate it appropriately, but do not maintain the posterior anchoring of the osk gene products.

\section{Role of F-actin in the localization of Osk, Bif, and Hom}

An intact F-actin cytoskeleton is thought to be required for asymmetric protein localization in several contexts (for review, see Jan and Jan 1998). In the oocyte, loss or reduction of the actin binding proteins moesin and tropomyosin have been shown to affect the posterior anchoring of Osk in the oocyte and the embryo, respectively (Erdelyi et al. 1995; Tetzlaff et al. 1996; Jankovics et al. 2002; Polesello et al. 2002). To assess the require- 


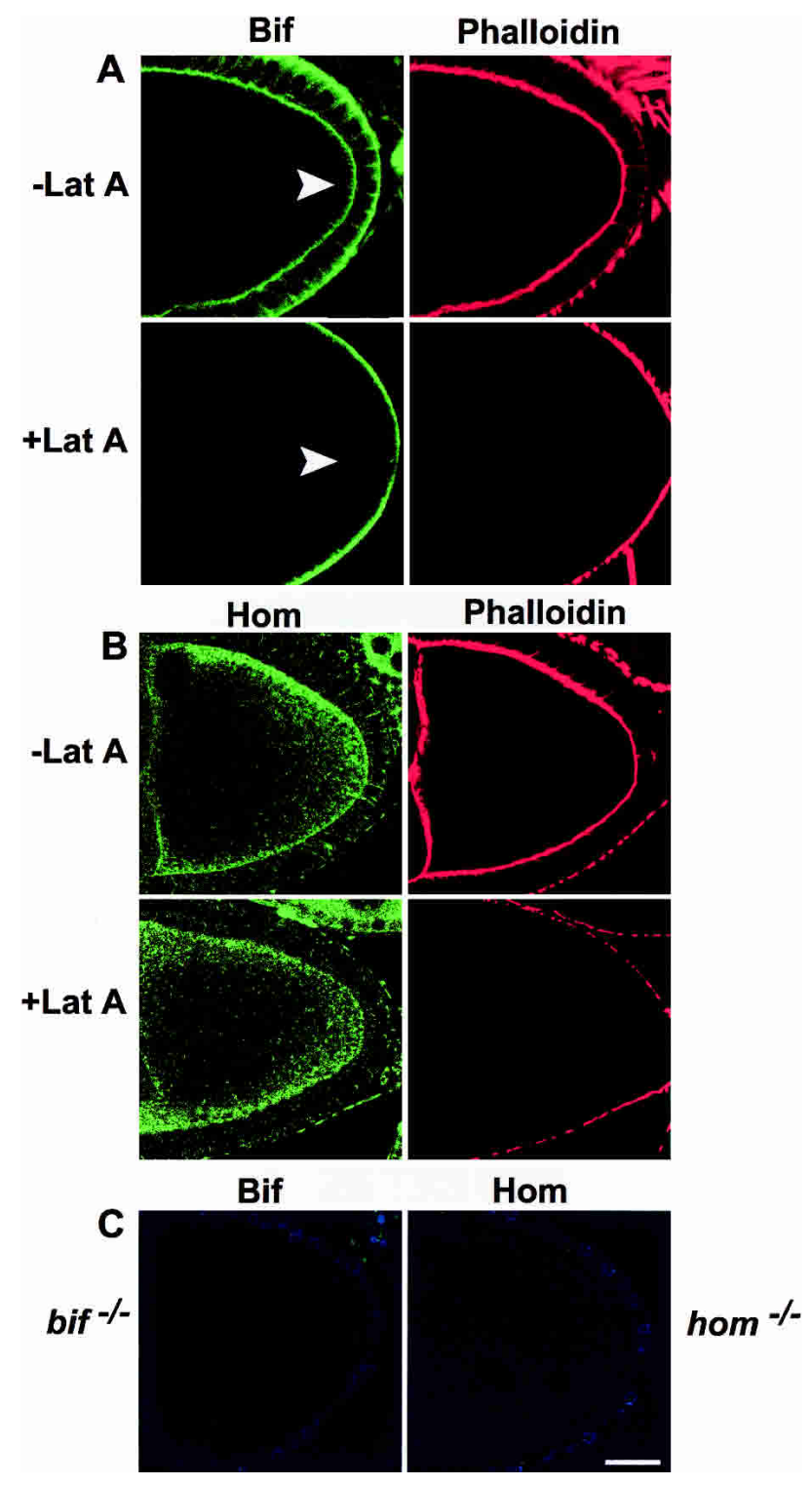

Figure 2. Bifocal and Homer localization in the presence and absence of intact microfilaments. (A) Anti-Bif (green) and phalloidin (red) staining of Lat A-treated and untreated wild-type oocytes. Note that cortical Bif staining (arrowheads) in the oocyte disappears in the absence of an intact F-actin cytoskeleton. (B) Anti-Hom (green) and phalloidin (red) staining in the presence and absence of Lat A treatment. Note that Hom staining at the cortex of the oocyte remains even in the absence of an intact F-actin cytoskeleton. $(C)$ Anti-Bif and anti-Hom staining of their respective mutant oocytes. In these oocytes the follicle cell nuclei are in blue. Note that in Lat A-treated ovaries, residual phalloidin staining remains on the exterior border of the follicle cells, whereas it is totally absent from the oocyte cortex, perhaps due to a lower actin turnover in the follicle cells.

ment for an intact microfilament cytoskeleton for the anchoring of osk RNA and proteins in the oocyte, the localization of these molecules was examined in wildtype oocytes treated with an actin depolymerizing drug, Latrunculin A (Lat A; Spector et al. 1983). Following treatment with $20 \mu \mathrm{m}$ Lat A, cortical F-actin in the oocytes was largely undetectable with phalloidin, yet, unexpectedly, both Osk proteins (short and Osk- $\beta$ Gal) and osk RNA remain localized to the posterior cortex of the great majority of wild-type oocytes from stage 9-10B (Fig. 3a-c; see Supplemental Table 1 for quantitation). This was seen even when the oocytes were overstained for the osk RNA: in around $17 \%$ of the Lat A-treated wild-type oocytes, mild defects in anchoring are observed. osk RNA and proteins show a diffuse localization at the posterior cortex. However, in no cases were they seen concentrated in the cytoplasm or had they become delocalized or undetectable (as seen in the hom/bif double mutant oocytes) as would be expected if an intact F-actin cytoskeleton were to be an absolute requirement for the normal anchoring of Osk. These mild effects on protein localization in the oocyte are in distinct contrast to those seen in embryonic neuroblasts where severe and high-penetrance defects in asymmetric protein localization are observed following disruption of microfilaments (Broadus and Doe 1997). These observations suggest that the role of microfilaments in the anchoring of proteins to the cortex may differ in the different cellular contexts.

Additional experiments were performed to ascertain whether the mild effects on Osk posterior anchoring following disruption of microfilaments are peculiar to Lat A treatment. In fact, the posterior cortical localization of Osk remained in the great majority of oocytes even after treatment with cytochalasin D (CD), Lat A followed by $\mathrm{CD}, \mathrm{CD}$ followed by Lat A, and up to $100 \mu \mathrm{M}$ Lat A. (Fig. $4 a-c$; data not shown; see figure legends for quantitation). These results are consistent with previous reports of CD disruption of F-actin, for example (Cha et al. 2002); however, they indicate that an intact F-actin cytoskeleton, although required for the normal posterior anchoring of Osk in a small proportion of oocytes, is probably not the only factor involved in normal anchoring of Osk to the posterior cortex. There are at least two possible explanations for these observations. First, a small amount of residual F-actin might remain even after sequential treatment with Lat A and CD, which is sufficient to anchor Osk normally in a small fraction of the drug-treated oocytes. Alternatively, there may be other factors besides an intact F-actin cytoskeleton, which can, in parallel, contribute toward the posterior anchoring of osk RNA and proteins.

We next assessed the requirement for intact microfilaments on Hom and Bif localization. Both Bif and Hom localize to the cortex (and in the case of Hom also the cytoplasm) of wild-type oocytes (Fig. 2A,B). Following depolymerization of F-actin with Lat A, such that cortical F-actin becomes undetectable in the oocyte, Hom localization appears unchanged from the wild-type pattern in all oocytes (Fig. 2B, $\mathrm{n}=49$ ), but Bif becomes highly diffuse with essentially no detectable enrichment at the cortex (Fig. 2A, n=55). This appears to be an effect on Bif localization and not stability, because the levels of the protein are not reduced as judged by Western blot analysis (data not shown). Treating oocytes with colchicine, which disrupts the microtubules, did not affect either Hom or Bif localization in the oocyte /data not shown). These findings raise the possibility that bif function might be dependent on intact F-actin; however, Hom localization is Lat A-insensitive, suggesting that its function in the oocyte may not require intact microfilaments. However, we cannot exclude the possibility that Lat A treatment allows for the retention of a small amount of the F-actin cytoskeleton, and this is stabilized in some way by Hom (see below). 
Hom, but not Bif, is required for Osk anchoring in the absence of an intact F-actin cytoskeleton

These data raise the possibility that there might be two processes, one that is microfilament-dependent and requires Bif, and another which is not dependent on intact microfilaments and requires Hom. Either process is sufficient to anchor the osk gene products to the posterior cortex of the great majority of the oocytes. One prediction of this hypothesis is that hom should be necessary to anchor posterior components in the absence of intact F-actin. Indeed, when hom single mutant oocytes were treated with Lat A, we found a large amount of cytoplasmic Osk at stage 9 and 10 near the posterior pole, and there was a large reduction in the Osk- $\beta$ Gal signal (Fig. $3 g-1$; Table 1). This could indicate that the loss of the longer Osk isoform may be the primary defect seen in Lat A-treated hom mutants, as this longer Osk isoform is known to be essential for osk RNA and protein anchoring (Vanzo and Ephrussi 2002). The defects induced by depolymerizing Factin in hom oocytes are similar to but more severe than those seen in bif;hom double mutant oocytes. This is probably due to the fact that Factin disruption also leads to premature streaming in stage 9 and enhanced streaming in stage 10 oocytes (Manseau et al. 1996), thus accentuating the effects of the loss of Osk anchoring at the posterior cortex.

The above results demonstrate that disruption of F-actin in the absence of hom function disrupts anchoring of the osk gene products. Similar results were obtained when hom mutants were treated with just CD or treated successively with Lat $A$ and $C D$ or vice versa (Fig. $4 \mathrm{~d}-\mathrm{f}$ ). CD does not cause loss of F-actin as seen with phalloidin staining, and causes changes in the cortical F-actin as well as causing some of the F-actin to be seen in the cytoplasm. This latter effect is not seen with Lat A. This could be attributed to the difference in the mechanism of action between CD and Lat A (Spector et al. 1989). However, despite this difference between $\mathrm{CD}$ and Lat A, the effects of these drugs singly or in combination on Osk posterior anchoring are similar, causing mild defects in Osk posterior anchoring in only onefifth of the treated wild-type oocytes and severe defects in the great majority of treated hom oocytes.

A second prediction is that disruption of microfilaments in the absence of bif should not affect anchoring of Osk. Indeed, most wild-type and bif single mutant oocytes treated with Lat A showed largely wild-type anchoring of the Osk gene products (see Table 1), similar to Lat A treatment of wild-type oocytes. These results are consistent with the notion that Bif functions in an F-actin-dependent manner in maintaining Osk to the posterior of the oocyte.

If there are two independent mechanisms which act redundantly for normal Osk anchoring at the posterior cortex, then it would follow that the bif; hom double mutants in the absence of an intact F-actin cytoskeleton would show a phenotype similar to that of hom single

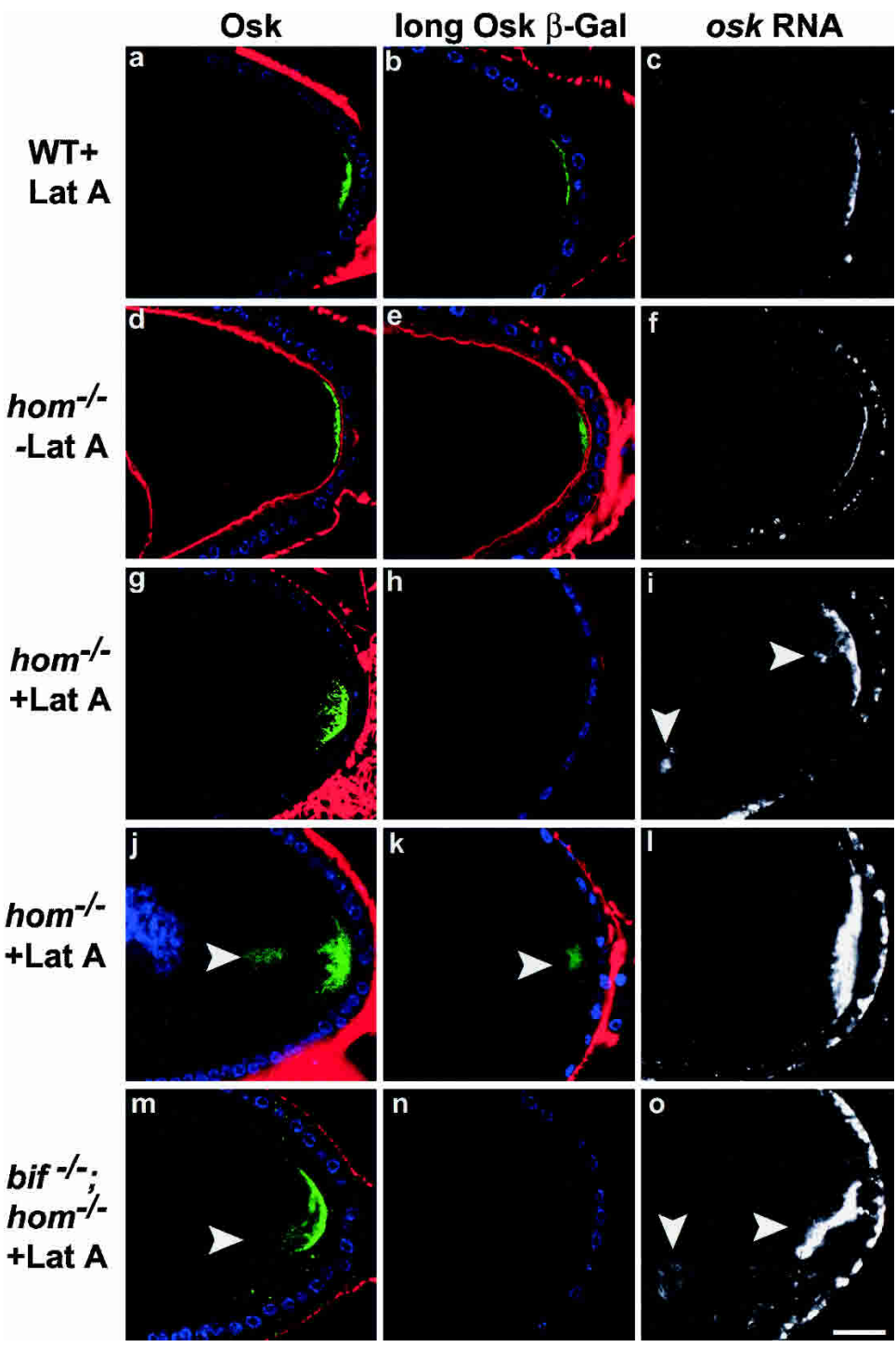

Figure 3. Osk protein and RNA localization in wild-type and hom oocytes in the presence or absence of intact microfilaments. In the great majority of wildtype stage-10 oocytes $(a-c)$, in which the cortical F-actin (phalloidin, red) has been rendered undetectable by Lat A treatment, Osk (green, $a$ ), Osk- $\beta$ Gal (green, $b$ ), and osk RNA (white, c) are localized as a tight posterior crescent similar to that of untreated wild-type oocytes (see Table 1 for quantitation). DNA staining shows the position of the follicle cell nuclei (blue). In hom oocytes not treated with Lat A (d-f), cortical F-actin (red) can be seen, and Osk protein $(d)$, Osk- $\beta$ Gal $(e)$, and osk RNA $(f)$ localizations are wild type. In Lat A-treated hom oocytes $(g-1)$, the cortical F-actin is undetectable. osk RNA and proteins show diffuse $(g, i, j, l)$ and in some cases prominent cytoplasmic localization (arrowheads, $i-k)$. In several cases the Osk- $\beta$ Gal line does not show any staining in hom mutants treated with Lat A (example in $h$ ). Similar results are seen with Lat A treatment of hom; bif double mutants ( $m-O$; cytoplasmic localization of Osk and osk RNA are indicated by arrowheads in $m, o$ ).

mutants treated with Lat A, and not a more severe phenotype. This is indeed the case we observed on testing osk RNA and Osk proteins in Lat A-treated double mutant oocytes (Fig. 3m-O; quantitation in Supplemental Table 1).

In light of the finding that Hom posterior cortical localization remains unchanged following F-actin disruption, its ability to localize Osk to the posterior may be because it forms a complex with Osk. Co-immunoprecipitation experiments, using Drosophila ovarian ex- 


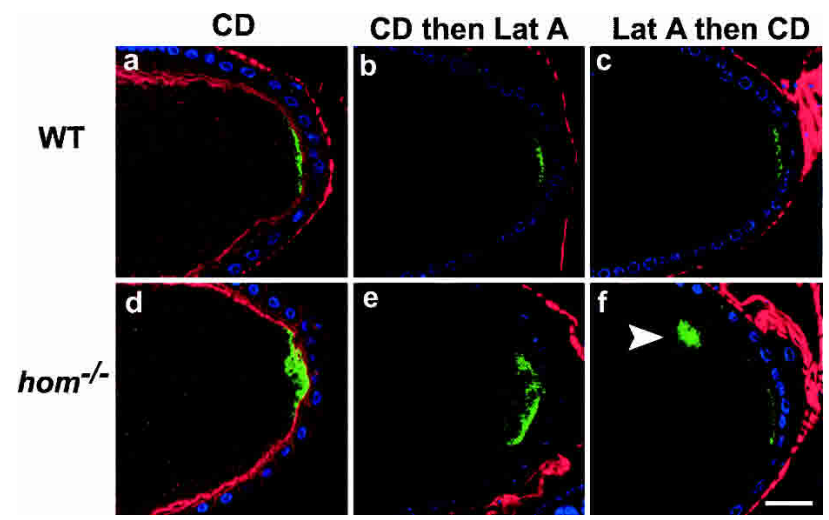

Figure 4. Osk localization in drug-treated wild-type and hom mutants and wild-type stage- 9 and -10 oocytes treated with CD alone (a: $\mathrm{n}=23,26 \%$ show diffuse staining, whereas the others are normal), CD followed by Lat A ( $b: \mathrm{n}=18,22 \%$ show diffuse staining), or Lat A followed by $\mathrm{CD}$ (c: $\mathrm{n}=15,20 \%$ show diffuse staining). Phalloidin staining in these oocytes is in red. Osk protein (green) in these oocytes was detected using anti-Osk antibody. The protein localization in most cases is still predominantly at the posterior cortex, as in the wild-type oocytes. Similar experiments with hom mutant oocytes treated with $C D$ alone $(d: 27.2 \%$ show diffuse staining; $45.5 \%$, cytoplasmic staining, $\mathrm{n}=11), \mathrm{CD}$ followed by Lat $\mathrm{A}$ (e: $22.2 \%$, diffuse staining; $16.7 \%$, no staining; $44.4 \%$, cytoplasmic staining, $\mathrm{n}=18)$, and Lat A followed by $\mathrm{CD}(f: 50 \%$, diffuse staining; $10 \%$, no stain; $30 \%$, cytoplasmic staining, $\mathrm{n}=10$ ) all show enhanced diffuse or cytoplasmic staining (arrowhead in $f$ ) of Osk after drug treatment. DNA staining (blue) shows the position of the follicle cell nuclei.

tracts, indicate that Hom and Osk form a complex in vivo. Further, the stability of this complex is not dependent on an intact F-actin cytoskeleton (Fig. 5A). We also tested the possibility that Hom protein may interact with osk RNA to give rise to defects seen in the mutants; however, no interaction was detected (data not shown).

\section{Model}

The maintenance of Osk at the posterior of the oocyte may be mediated by two distinct mechanisms, either of which is sufficient, at least for the great majority of the oocytes. One mechanism does not require an intact Factin cytoskeleton, and Hom seems to be an important player in this process. Hom can complex with Osk, and the stability of this complex is not dependent on an intact F-actin cytoskeleton. The second mechanism requires an intact F-actin cytoskeleton. Bif seems to be required for this mechanism. Previous work has shown that overexpression of Bif can promote actin polymerization in cultured cells (Ruan et al. 2002). As it is also known that F-actin forms a complex with Bif in Drosophila embryonic lysates (Sisson et al. 2000) and that Bif binds directly to F-actin filaments in vitro (data not shown), it is possible that Bif acts to stabilize actin filaments. In this scenario its absence may cause subtle changes in the F-actin cytoskeleton that may affect its capacity to anchor molecules at the cortex when hom is absent. In contrast, hom can function and is required to anchor Osk in the absence of an intact F-actin cytoskeleton or in the absence of bif function. Only when both mechanisms are disrupted in the oocyte, either through the simultaneous disruption of both hom and bif, or when F-actin is disrupted in the absence of hom, do the osk gene products fail to remain anchored to the posterior cortex (schematically represented in Fig. 5B).

Recent studies showed that Drosophila moesin is essential to link the cortical F-actin to the oocyte cell membrane (Jankovics et al. 2002; Polesello et al. 2002). When moesin function is compromised, cortical F-actin can detach from the cell membrane and "fall" into the oocyte cytoplasm, and this results in the mislocalization of Osk. We have examined the effects of loss of moesin function on the localization of both Bif and Hom; our results (K. Babu, unpubl.) indicate that in these mutant oocytes, where the cortical F-actin detaches from the mutant oocyte cell membrane, components of both of our proposed anchoring pathways, Hom and Bif, also detach. These observations are consistent both with the Osk mislocalization phenotype seen in moesin mutant oocytes and with our model. It will be interesting to identify additional molecules involved in these separate pathways and to elucidate the mechanisms that are required to localize Hom to the posterior cortex of the oocyte in the absence of an intact actin cytoskeleton.

\section{Materials and Methods}

Additional details are provided in Supplementary Materials.

\section{Fly strains}

Canton S was used as wild-type control. bifR47 (Bahri et al. 1997) and homLL17 were antigen minus alleles used for our studies. khc $\beta$-Gal

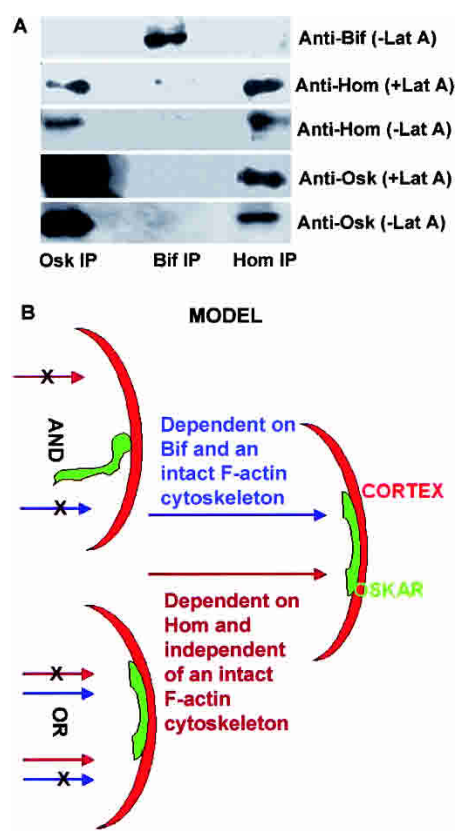

Figure 5. Immunoprecipitations and proposed model. (A) Western blot analysis of co-immunoprecipitations (co-IPs) from extracts of ovaries treated or not treated with Lat A. Anti-Osk, anti-Bif, and anti-Hom co-IPs were probed with the antibodies indicated. Osk appears to complex with Hom in extracts derived from Lat A-treated and untreated ovaries. Bif does not co-IP with Osk or Hom and serves as a control. Note that the amount of Osk brought down in the Osk IP with Lat A is similar to the amount of Osk brought down in the absence of Lat A. (B) A schematic model proposing that the posterior cortical anchoring of the osk gene products in the oocyte is mediated by an F-actin-dependent pathway requiring bif and a second parallel pathway requiring hom which does not require intact F-actin; either pathway can promote anchoring of the osk gene products at the posterior cortex of the great majority of oocytes. Posterior is toward the right. 
(Brendza et al. 2000) and Osk- $\beta$ Gal transgenes (Vanzo and Ephrussi 2002) were previously described.

Fish and immunoflourescence in ovaries

FISH analysis (Vanzo and Ephrussi 2002) and antibody stainings (Tomancak et al. 2000) were performed as previously described.

Drug treatment

Details of ovary dissections and treatment with Lat A and cytochalasin D are provided in Supplementary Materials.

Immunoprecipitation experiments and Western blotting Preparation of ovarian lysates, immunoprecipitations, and Western blotting was performed using standard protocols.

\section{Acknowledgments}

We thank P. Cohen, A. Ephrussi, T. Karr, P. Lasko, F. Payre, W. Saxton, G. Schupback, and D. St Johnston for reagents; A. Ephrussi and D. St. Johnston for advice; P. Overton and R. Tuxworth for critical reading of the manuscript; and $\mathrm{R}$. Tuxworth for help with image collection. We thank Fengwei Yu for help with the generation of the anti-Homer antibodies, and the Bloomington Stock Center for fly stocks. We thank the members of the Chia, Tear, and Yang Labs for help and suggestions. W.C. is a Wellcome Trust Principal Research Fellow. The Wellcome Trust and IMCB (A-STAR) Singapore provided support.

The publication costs of this article were defrayed in part by payment of page charges. This article must therefore be hereby marked "advertisement" in accordance with 18 USC section 1734 solely to indicate this fact.

\section{References}

Bahri, S.M., Yang, X., and Chia, W. 1997. The Drosophila bifocal gene encodes a novel protein which colocalizes with actin and is necessary for photoreceptor morphogenesis. Mol. Cell Biol. 17: 5521-5529.

Berleth, T., Burri, M., Thoma, G., Bopp, D., Richstein, S., Frigerio, G., Noll, M., and Nusslein-Volhard, C. 1988. The role of localization of bicoid RNA in organizing the anterior pattern of the Drosophila embryo. EMBO J. 7: 1749-1756.

Brakeman, P.R., Lanahan, A.A., O'Brien, R., Roche, K., Barnes, C.A., Huganir, R.L., and Worley, P.F. 1997. Homer: A protein that selectively binds metabotropic glutamate receptors. Nature 386: 284288.

Brendza, R.P., Serbus, L.R., Duffy, J.B., and Saxton, W.M. 2000. A function for kinesin I in the posterior transport of oskar mRNA and Staufen protein. Science 289: 2120-2122.

Broadus, J. and Doe, C.Q. 1997. Extrinsic cues, intrinsic cues and microfilaments regulate asymmetric protein localization in Drosophila neuroblasts. Curr. Biol. 7: 827-835.

Cha, B.J., Serbus, L.R., Koppetsch, B.S., and Theurkauf, W.E. 2002. Kinesin I-dependent cortical exclusion restricts pole plasm to the oocyte posterior. Nat. Cell Biol. 4: 592-598.

Clark, I., Giniger, E., Ruohola-Baker, H., Jan, L.Y., and Jan, Y.N. 1994. Transient posterior localization of a kinesin fusion protein reflects anteroposterior polarity of the Drosophila oocyte. Curr. Biol. 4: 289300.

Diagana, T.T., Thomas, U., Prokopenko, S.N., Xiao, B., Worley, P.F., and Thomas, J.B. 2002. Mutation of Drosophila homer disrupts control of locomotor activity and behavioral plasticity. J. Neurosci. 22: 428436.

Ephrussi, A. and Lehmann, R. 1992. Induction of germ cell formation by oskar. Nature 358: 387-392.

Ephrussi, A., Dickinson, L.K., and Lehmann, R. 1991. Oskar organizes the germ plasm and directs localization of the posterior determinant nanos. Cell 66: 37-50.

Erdelyi, M., Michon, A.M., Guichet, A., Glotzer, J.B., and Ephrussi, A. 1995. Requirement for Drosophila cytoplasmic tropomyosin in oskar mRNA localization. Nature 377: 524-527.

Glotzer, J.B., Saffrich, R., Glotzer, M., and Ephrussi, A. 1997. Cytoplasmic flows localize injected oskar RNA in Drosophila oocytes. Curr. Biol. 7: 326-337.

Graner, M., Stupka, K., and Karr, T.L. 1994. Biochemical and cytological characterization of DROP-1: A widely distributed proteoglycan in Drosophila. Insect Biochem. Mol. Biol. 24: 557-567.

Gunkel, N., Yano, T., Markussen, F.H., Olsen, L.C., and Ephrussi, A. 1998. Localization-dependent translation requires a functional interaction between the $5^{\prime}$ and $3^{\prime}$ ends of oskar mRNA. Genes \& Dev. 12: $1652-1664$.

Helps, N.R., Cohen, P.T., Bahri, S.M., Chia, W., and Babu, K. 2001. Interaction with protein phosphatase 1 is essential for bifocal function during the morphogenesis of the Drosophila compound eye. Mol. Cell Biol. 21: 2154-2164.

Jan, Y.N. and Jan, L.Y. 1998. Asymmetric cell division. Nature 392: 775 778.

Jankovics, F., Sinka, R., Lukacsovich, T., and Erdelyi, M. 2002. MOESIN crosslinks actin and cell membrane in Drosophila oocytes and is required for oskar anchoring. Curr. Biol. 12: 2060-2065.

Karr, T.L. 1991. Intracellular sperm/egg interactions in Drosophila: A three-dimensional structural analysis of a paternal product in the developing egg. Mech. Dev. 34: 101-111.

Kim-Ha, J., Smith, J.L., and Macdonald, P.M. 1991. oskar mRNA is localized to the posterior pole of the Drosophila oocyte. Cell 66: 23-35.

Lehmann, R. and Nusslein-Volhard, C. 1986. Abdominal segmentation, pole cell formation, and embryonic polarity require the localized activity of oskar, a maternal gene in Drosophila. Cell 47: 141-152.

Manseau, L., Calley, J., and Phan, H. 1996. Profilin is required for posterior patterning of the Drosophila oocyte. Development 122: 2109-2116.

Neuman-Silberberg, F.S. and Schupbach, T. 1996. The Drosophila TGF$\alpha$-like protein Gurken: Expression and cellular localization during Drosophila oogenesis. Mech. Dev. 59: 105-113.

Polesello, C., Delon, I., Valenti, P., Ferrer, P., and Payre, F. 2002. Dmoesin controls actin-based cell shape and polarity during Drosophila melanogaster oogenesis. Nat. Cell Biol. 4: 782-789.

Rongo, C., Gavis, E.R., and Lehmann, R. 1995. Localization of oskar RNA regulates oskar translation and requires Oskar protein. Development 121: 2737-2746.

Ruan, W., Long, H., Vuong, D.H., and Rao, Y. 2002. Bifocal is a downstream target of the Ste20-like serine/threonine kinase misshapen in regulating photoreceptor growth cone targeting in Drosophila. Neuron 36: 831-842.

Saxton, W.M. 2001. Microtubules, motors, and mRNA localization mechanisms: Watching fluorescent messages move. Cell 107: 707710.

Shiraishi, Y., Mizutani, A., Bito, H., Fujisawa, K., Narumiya, S., Mikoshiba, K., and Furuichi, T. 1999. Cupidin, an isoform of Homer/Vesl, interacts with the actin cytoskeleton and activated rho family small GTPases and is expressed in developing mouse cerebellar granule cells. J. Neurosci. 19: 8389-8400.

Sisson, J.C., Field, C., Ventura, R., Royou, A., and Sullivan, W. 2000. Lava lamp, a novel peripheral golgi protein, is required for Drosophila melanogaster cellularization. J. Cell Biol. 151: 905-918.

Spector, I., Shochet, N.R., Kashman, Y., and Groweiss, A. 1983. Latrunculins: Novel marine toxins that disrupt microfilament organization in cultured cells. Science 219: 493-495.

Spector, I., Shochet, N.R., Blasberger, D., and Kashman, Y. 1989. Latrunculins-Novel marine macrolides that disrupt microfilament organization and affect cell growth: I. Comparison with cytochalasin D. Cell Motil. Cytoskeleton 13: 127-144.

St Johnston, D., Beuchle, D., and Nusslein-Volhard, C. 1991. Staufen, a gene required to localize maternal RNAs in the Drosophila egg. Cell 66: $51-63$.

Tetzlaff, M.T., Jackle, H., and Pankratz, M.J. 1996. Lack of Drosophila cytoskeletal tropomyosin affects head morphogenesis and the accumulation of oskar mRNA required for germ cell formation. EMBO $J$. 15: $1247-1254$.

Theurkauf, W.E. 1994. Premature microtubule-dependent cytoplasmic streaming in cappuccino and spire mutant oocytes. Science 265: 2093-2096.

Tomancak, P., Piano, F., Riechmann, V., Gunsalus, K.C., Kemphues, K.J., and Ephrussi, A. 2000. A Drosophila melanogaster homologue of Caenorhabditis elegans par- 1 acts at an early step in embryonic-axis formation. Nat. Cell Biol. 2: 458-460.

Vanzo, N.F. and Ephrussi, A. 2002. Oskar anchoring restricts pole plasm formation to the posterior of the Drosophila oocyte. Development 129: $3705-3714$. 


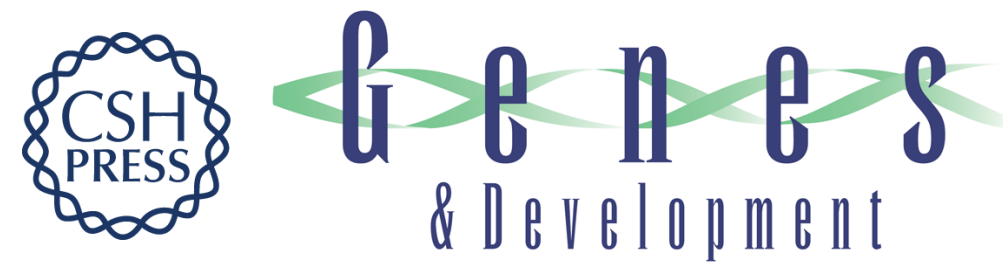

\section{Roles of Bifocal, Homer, and F-actin in anchoring Oskar to the posterior cortex of Drosophila oocytes}

Kavita Babu, Yu Cai, Sami Bahri, et al.

Genes Dev. 2004, 18:

Access the most recent version at doi:10.1101/gad.282604

Supplemental
Material http://genesdev.cshlp.org/content/suppl/2004/01/08/18.2.138.DC1

References This article cites 34 articles, 12 of which can be accessed free at: http://genesdev.cshlp.org/content/18/2/138.full.html\#ref-list-1

License

Email Alerting

Receive free email alerts when new articles cite this article - sign up in the box at the top Service right corner of the article or click here.

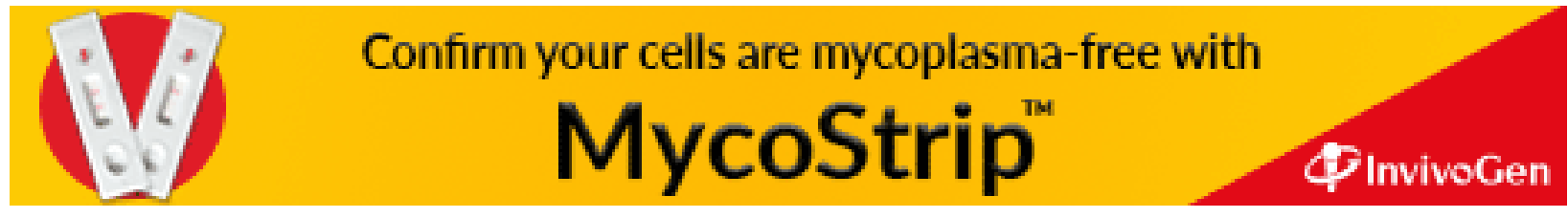

